\title{
Integration of Irreversible Gates in Reversible Circuits Using NCT Library
}

\author{
Mohamed Osman ${ }^{1}$, Ahmed Younes ${ }^{2}$, Moustafa H.Fahmy ${ }^{2}$ \\ ${ }^{1}$ (Mathematics and Computer Science, Faculty of Science / Damanhour University, Egypt) \\ ${ }^{2}$ (Mathematics and Computer Science, Faculty of Science / Alexandria University, Egypt)
}

\begin{abstract}
The reversible circuit synthesis problem can be reduced to permutation group. This allows Schreier-Sims Algorithm for the strong generating set-finding problem to be used to synthesize reversible circuits using the NCT library. Applying novel optimization rules to minimize the number of gates gives better quantum cost than that shown in the literature. Applications on how to integrate any three irreversible Boolean functions on a single 3-bit reversible circuit will be shown.
\end{abstract}

Keywords: Reversible Circuit, Quantum Cost, Circuit Optimization, Group Theory

\section{INTRODUCTION}

Reversible logic $[1,2]$ is an active area of research. It has many applications in quantum computing $[3,4]$, low-power CMOS $[5,6]$ and many more. Synthesis and optimization of Boolean reversible circuits cannot be done using classical methods [7]. Optimization of Boolean systems on non-standard computers that promise to do computation more powerfully [8] than classical computers, such as quantum computers, is essential to explore the novel applications that can be applied on such systems.

The study of reversible logic synthesis problem using group theory is arising rapidly. Investigation on the universality of the basic building blocks of reversible circuits has been done [9,10]. A relation between Young subgroups and the reversible logic synthesis problem has been proposed [11]. A comparison between the decomposition of reversible circuit and quantum circuit using group theory has been shown [12]. GAP-based algorithms that synthesize reversible circuits for various types of gate with various gate costs have been proposed [13]. Tight bounds on the synthesis of 3-bit reversible circuits using NCT library has been shown in [14].

The first aim of the paper is to synthesize 3-bit reversible circuits using NCT library with better quantum cost than that shown in the literature. The second aim is to use the synthesized circuits to design a reversible circuit that simulates the function of irreversible Boolean functions such as $A N D, O R, N O T$, $X O R, N A N D$ and $N O R$ in a single zero-garbage reversible circuit by integrating any three Boolean functions as long as this integration keep the reversibility of the circuit.

The paper is organized as follows: Sect. 2 gives a short background on the synthesis of reversible circuit problem and shows the reduction the problem to permutation group. Sect. 3 shows the optimization rules applied on reversible circuits obtained using Schreier-Sims Algorithm for the strong generating set-finding problem [15] to decrease the quantum cost of the circuits. Sect. 4 shows the results of the experiments. Sect. 5 shows the results of integrating irreversible Boolean functions in a single 3-bit reversible circuit. The paper ends up with a summary and conclusion in Sect. 6 .

\section{BACKGROUND}

This section will review the basic definitions of reversible circuits, the definition of quantum cost of reversible circuits and the basic notions for reversible circuit synthesis, the relationship between reversible logic circuits and permutation group theory.

\subsection{BASIC DEFINITIONS}

Definition 1: Let $X=\{0,1\}$. A Boolean function $f$ with $n$ input variables $x_{1}, \ldots, x_{n}$ and $n$ output variables $y_{1}, \ldots, y_{n}$, is a function $f: X^{n} \rightarrow X^{n}$, where $\left(x_{1}, \ldots, x_{n}\right) \in X^{n}$ is called the input vector and $\left(y_{1}, \ldots, y_{n}\right) \in X^{n}$ is called the output vector.

Definition 2: An $n$-input $n$-output Boolean function is reversible $(n \times n$ function) if it maps each input vector to a unique output vector, i.e. a one-to-one, onto function (bijection). There are $2^{n} !$ reversible $n \times n$ Boolean functions. For $n=3$, there are 403203 -in/out reversible functions.

Definition 3: An $n$-input $n$-output ( $n$-in/out) reversible gate (or circuit) is a gate that realizes a $n \times n$ reversible function. 
Definition 4: When an $m$-in/out reversible gate $U$ is applied on an $n$-in/out reversible circuit such that $m \leq n$, then $U$ will be denoted as $U_{i 1 i 2 \ldots i m}^{n}$ where $\left\{i_{1}, i_{2}, \ldots, i_{m}\right\}$ are the $m$ wires spanned by $U$ in order. library $L$.

Definition 5: A set of reversible gates that can be used to build a reversible circuit is called a gate

Definition 6: A universal reversible gate library $L_{n}$ is a set of reversible gates such that a cascading of gates in $L_{n}$ can be used to synthesize any reversible circuit with $n$-in/out.

Definition 7: A universal reversible gate sub library $S L_{n}$ is a set of reversible gates such that $S L_{n} \subseteq L_{n}$ that can be used to build any reversible circuit with $n$-in/out.

Definition 8: Let a finite set $A=\{1,2, \ldots, N\}$ and a bijection $\delta: A \rightarrow A$, then $\delta$ can be written as,

$$
\left(\begin{array}{ccccc}
1 & 2 & 3 & \ldots & n \\
\delta(1) & \delta(2) & \delta(3) & \ldots & \delta(n)
\end{array}\right)
$$

i.e. $\delta$ is a permutation of A. Let $A$ be an ordered set, then the top row can be eliminated and $\delta$ can be written as,

$$
(\delta(1), \quad \delta(2), \delta(3), \ldots, \delta(n))
$$

Any reversible circuit with $\mathrm{n}$-in/out can be considered as a permutation $\delta$ and (2) is called the specification of this reversible circuit such that $N=2^{n}$.

The set of all permutations on $A$ forms a symmetric group on $A$ under composition of mappings [16], denoted by $S_{N}$ [17]. A permutation group $G$ is a subgroup of the symmetric group $S_{N}$ [16]. A universal reversible gate library $L_{n}$ is called the generators of the group. Another important notation of a permutation is the product of disjoint cycles [17]. For example, $\left(\begin{array}{l}1,2,3,4,5,6,7,8 \\ 1,2,4,3,7,6,8,5\end{array}\right)$ will be written as $(3,4)(5,7,8)$. The identity mapping "()" is called the unit element in a permutation group. A product $p * q$ of two permutations $p$ and $q$ means applying mapping $p$ then $q$, which is equivalent to cascading $p$ and $q$.

\subsection{ReVERSIBLE CirCuITS}

The $C^{n} N O T$ gate is used as the main reversible gate to build any reversible circuit, since it is proved to be universal for reversible logic synthesis [7]. The $C^{n} N O T$ gate is defined as shown in Fig.1.

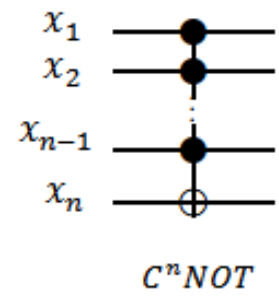

Figure 1: $C^{n}$ NOT gate. The control bit line is denoted by $\bullet$, and the target bit line is denoted by $\oplus$.

The action of $C^{n} N O T$ gate is defined as follows, if the control bit lines are set to 1 then the target bit line is flipped, otherwise the target bit line is left unchanged. Some special cases of the $C^{n} N O T$ gate are defined as follows, $C^{1} N O T$ gate with no control bit is called NOT gate. $C^{2} N O T$ with one control bit is called CNOT gate. $C^{3} N O T$ with two control bits is called Toffoli gate. For the sake of readability $C^{1} N O T, C^{2} N O T$ and $C^{3} N O T$ will be written shortly as $N, C$ and $T$ respectively where the control and/or target bits will be shown in the subscript of the gate and the total number of bits will be shown in the superscript. The $N, C$ and $T$ gates can be used to form a universal library for 3-in/out reversible circuits known as NCT library. The main NCT library consists of 12 gates as shown in Fig.2. 


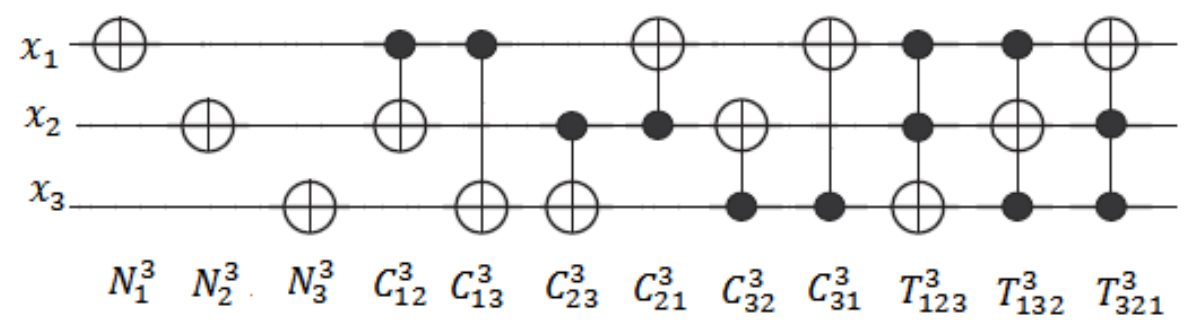

Figure 2: The main NCT library consists of 12 gates

A gate library with $N$ gates is not universal for 3-in/out reversible circuits since it can realize only 8 possible circuits from the 40320 circuits [18]. For $n$-in/out reversible circuits, there are n possible $N$ gates. There are 3 possible $N$ gates as shown in Fig.3.

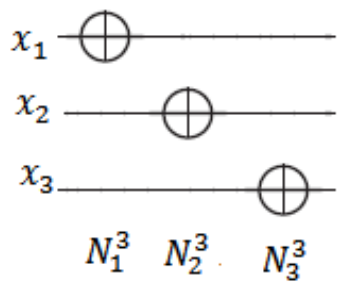

Figure 3: The 3 possible $N$ gates for 3-bit reversible circuits.

$$
\begin{aligned}
& N_{1}^{3}:\left(x_{1}, x_{2}, x_{3}\right) \stackrel{\text { yields }}{\longrightarrow}\left(x_{1} \oplus 1, x_{2}, x_{3}\right) \equiv(1,5)(2,6)(3,7)(4,8), \\
& N_{2}^{3}:\left(x_{1}, x_{2}, x_{3}\right) \stackrel{\text { yield }}{\longrightarrow}\left(x_{1}, x_{2} \oplus 1, x_{3}\right) \equiv(1,3)(2,4)(5,7)(6,8), \\
& N_{3}^{3}:\left(x_{1}, x_{2}, x_{3}\right) \stackrel{\text { yields }}{\longrightarrow}\left(x_{1}, x_{2}, x_{3} \oplus 1\right) \equiv(1,2)(3,4)(5,6)(7,8) .
\end{aligned}
$$

A gate library with $C$ gates can realize a total of 168 reversible circuits [18]. There are 6 possible $C$ gates for the 3-in/out reversible circuits as shown in Fig.4.

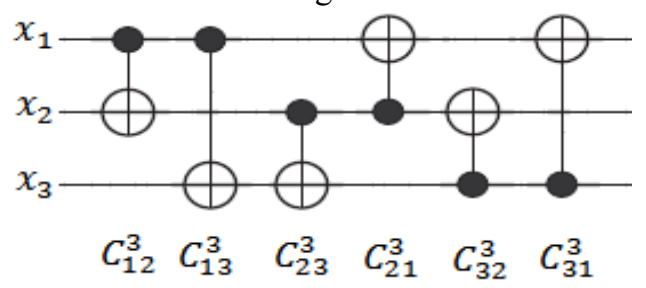

Figure 4: The 6 possible $C$ gates for 3-bit reversible circuits.

$$
\begin{aligned}
& C_{12}^{3}:\left(x_{1}, x_{2}, x_{3}\right) \stackrel{\text { yields }}{\longrightarrow}\left(x_{1}, x_{2} \oplus x_{1}, x_{3}\right) \equiv(5,7)(6,8), \\
& C_{13}^{3}:\left(x_{1}, x_{2}, x_{3}\right) \stackrel{\text { yields }}{\longrightarrow}\left(x_{1}, x_{2}, x_{3} \oplus x_{1}\right) \equiv(5,6)(7,8), \\
& C_{23}^{3}:\left(x_{1}, x_{2}, x_{3}\right) \stackrel{\text { yields }}{\longrightarrow}\left(x_{1}, x_{2}, x_{3} \oplus x_{2}\right) \equiv(3,4)(7,8), \\
& C_{21}^{3}:\left(x_{1}, x_{2}, x_{3}\right) \stackrel{\text { yields }}{\longrightarrow}\left(x_{1} \oplus x_{2}, x_{2}, x_{3}\right) \equiv(3,7)(4,8), \\
& C_{32}^{3}:\left(x_{1}, x_{2}, x_{3}\right) \stackrel{\text { yields }}{\longrightarrow}\left(x_{1}, x_{2} \oplus x_{3}, x_{3}\right) \equiv(2,4)(6,8), \\
& C_{31}^{3}:\left(x_{1}, x_{2}, x_{3}\right) \stackrel{\text { yields }}{\longrightarrow}\left(x_{1} \oplus x_{3}, x_{2}, x_{3}\right) \equiv(2,6)(4,8) .
\end{aligned}
$$

The $T$ gate is the smallest reversible gate that is proved to be universal for non-reversible computation as it is proved to function as $N A N D$ gate by initializing the target bit to 1 [7]. A gate library with $T$ gate is not universal for reversible computation since it can realize only 24 possible 3 -in/out reversible circuits [18]. There are three possible $T$ gates for the 3-in/out reversible circuits as shown in Fig.5. 


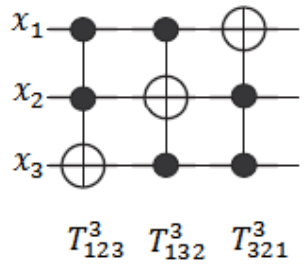

Figure 5: The 3 possible Toffoli (T) gates for 3-bit reversible circuits.

$$
\begin{aligned}
& T_{123}^{3}:\left(x_{1}, x_{2}, x_{3}\right) \stackrel{\text { yields }}{\longrightarrow}\left(x_{1}, x_{2}, x_{3} \oplus x_{1} x_{2}\right) \equiv(7,8), \\
& T_{132}^{3}:\left(x_{1}, x_{2}, x_{3}\right) \stackrel{\text { yields }}{\longrightarrow}\left(x_{1}, x_{2} \oplus x_{1} x_{3}, x_{3}\right) \equiv(6,8), \\
& T_{321}^{3}:\left(x_{1}, x_{2}, x_{3}\right) \stackrel{\text { yields }}{\longrightarrow}\left(x_{1} \oplus x_{2} x_{3}, x_{2}, x_{3}\right) \equiv(4,8) .
\end{aligned}
$$

For 3-bits reversible circuits, there are 40320 possible 3-in/out reversible circuits. The $N$ gate, the $C$ gate and the $T$ gate (NCT library) can used to synthesize all 40320 possible 3 -in/out reversible circuits.

\subsection{QUANTUM COST}

The quantum cost of a reversible circuit refers to optimization measurement as well as the number of $C^{n} N O T$ gates used in the circuit. The quantum cost of a reversible circuit is measured by the number of elementary gates required to build the $C^{n} N O T$ gate [19], i.e. the number of 2-qubit gates used in its implementation as a quantum circuit. In this paper, the cost of $N$ gate is ignored as in [13] to be able to compare results, i.e. the cost of $N$ is equal zero, and the cost of any 2-qubit gate is 1 and the quantum cost of $T$ is equal 5 as shown in Fig.6.

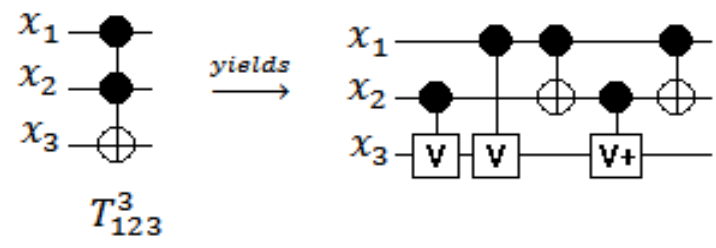

Figure 6: Decomposition of a $T$ gate as 5 elementary gates.

When implementing a reversible circuit, there are four elementary quantum gates that will be used: $N$ gate, $C$ gate, Controlled- $V$ and Controlled- $V^{+}$gates, where $V V^{+}=V^{+} V=I, V V=V^{+} V^{+}=N$, and $I$ is the identity gate [19].

\section{OPTIMIZATION RULES TO REDUCE QUANTUM COST}

Optimization rules will be used to identify and classify similarity of gates among a circuit when decomposed to a sequence of quantum gates. Decomposition of 3-bit reversible circuits can be used to decrease the quantum cost. Optimization is done by removing and/or combining (merging) adjacent gates act on the same qubit lines [20]. For example, the cost of the sequence of reversible gates $\left[T_{123}^{3}, C_{12}^{3}\right]$ is 4 instead of 6 as shown in Fig.7, the cost of the sequence of reversible gates $\left[T_{123}^{3}, C_{21}^{3}\right]$ is 5 instead of 6 as shown in Fig. 8 and the cost of the sequence of reversible gates $\left[T_{321}^{3}, T_{132}^{3}\right]$ is 9 instead of 10 , because the sequence of gate $C_{32}^{3}$ and gate $V_{32}^{3}$ can be combined as one gate $\left[C_{32}^{3} V_{32}^{3}\right]$ [20] as shown in Fig.9. The optimization rules used in this paper to decrease the quantum cost of the 3 -bits reversible circuits are shown in Table 1.
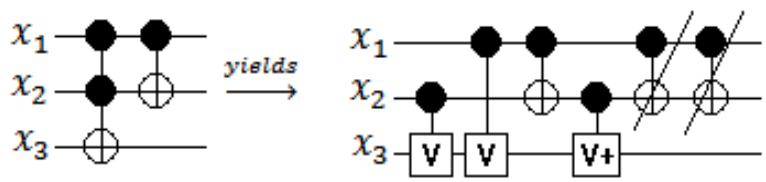

Figure 7: Decomposition of 3-bit reversible circuit $\left[T_{123}^{3}, C_{12}^{3}\right]$ as 4 elementary gates.
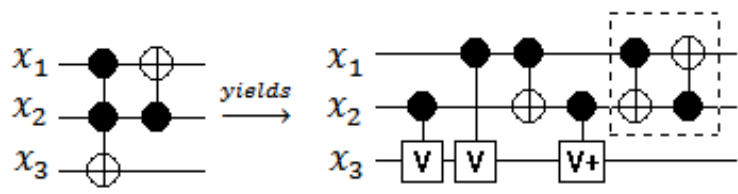

Figure 8: Decomposition of 3-bit reversible circuit $\left[T_{123}^{3}, C_{21}^{3}\right]$ as 5 elementary gates. 

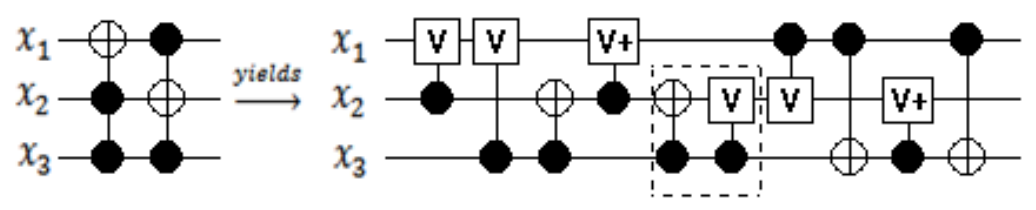

Figure 9: Decomposition of 3-bit reversible circuit $\left[T_{321}^{3}, T_{132}^{3}\right]$ as 9 elementary gates.

Table 1: Comparison of quantum cost using the proposed optimization rules and the existing work [21].

\begin{tabular}{|c|c|c|}
\hline \multirow{2}{*}{$N C T$ Circuits } & \multicolumn{2}{|c|}{ Quantum Cost } \\
\cline { 2 - 3 } & {$[21]$} & $\begin{array}{c}\text { Proposed } \\
\text { rules }\end{array}$ \\
\hline$T_{123}^{3} C_{12}^{3}$ & 6 & 4 \\
\hline$T_{123}^{3} C_{21}^{3}$ & 6 & 5 \\
\hline$T_{132}^{3} C_{13}^{3}$ & 6 & 4 \\
\hline$T_{132}^{3} C_{31}^{3}$ & 6 & 5 \\
\hline$T_{321}^{3} C_{23}^{3}$ & 6 & 5 \\
\hline$T_{321}^{3} C_{32}^{3}$ & 6 & 4 \\
\hline$T_{321}^{3} T_{132}^{3}$ & 10 & 9 \\
\hline$T_{123}^{3} T_{132}^{3}$ & 10 & 9 \\
\hline$T_{132}^{3} T_{123}^{3}$ & 10 & 9 \\
\hline
\end{tabular}

\section{EXPERIMENTAL RESULTS}

Experiments on 3-bits reversible circuits' synthesis are presented using the NCT library, the SchreierSims algorithm [14] is implemented using the group-theory algebraic software GAP [22]. This algorithm deals with minimal number of generator problems, which is more reasonable in practice because the gates have different costs. Some papers discussed these problems by other methods such as [13] and [14] but the SchreierSims algorithm realized in GAP [22] gives the similar minimal length problems result as shown in [13] and [14] and better results to minimize the quantum cost by applying the optimization rules as will be shown later.

Table 2 and Table 3 show the results for the main NCT gate library as a universal reversible library for reversible computation since it can realize the 40320 possible 3 -in/out reversible circuits. Table 3 gives the same result as Table 2 with more details on the cost of minimum length. The main NCT gate library synthesizes the best maximum length circuits, where the best maximum length $=8$ gates with $\operatorname{cost}=21$ and maximum $\operatorname{cost}=25$ with circuit length $=7$. Using group-theory algebraic software GAP [22] shows that the average length of a reversible circuit synthesized with NCT library is 5.865 which is similar to that shown in [13] and [14].

Table 4 and Table 5 show the results for the sub-libraries of the main NCT gate library which synthesize the best maximum cost circuits, where the best maximum cost $=17$ with circuit length $=7$ and maximum length $=12$ with cost $=14$. Table 5 gives the same result as Table 4 with more details on the length of minimum cost. Using group-theory algebraic software GAP [22] and apply the optimization rules to calculate the quantum cost of reversible circuits show that the average cost of reversible circuits synthesized with NCT library is 11.459 .

Table 2: Number of circuits with minimum length

\begin{tabular}{|c|c|c|c|}
\hline $\begin{array}{l}\text { Mini- } \\
\text { length }\end{array}$ & $\begin{array}{l}N C T- \\
\text { Circuits }\end{array}$ & $\begin{array}{l}\text { NCT- } \\
\text { Circuits[14] }\end{array}$ & $\begin{array}{l}\text { NCT- } \\
\text { Circuits[13] }\end{array}$ \\
\hline 0 & 1 & 1 & 1 \\
\hline 1 & 12 & 12 & 12 \\
\hline 2 & 102 & 102 & 102 \\
\hline 3 & 625 & 625 & 625 \\
\hline 4 & 2780 & 2780 & 2780 \\
\hline 5 & 8921 & 8921 & 8921 \\
\hline 6 & 17049 & 17049 & 17049 \\
\hline 7 & 10253 & 10253 & 10253 \\
\hline 8 & 577 & 577 & 577 \\
\hline Total & 40320 & 40320 & 40320 \\
\hline Average & 5.865 & 5.865 & 5.865 \\
\hline
\end{tabular}


Table 4: Number of circuits with minimum cost.

\begin{tabular}{|c|c|c|c|}
\hline $\begin{array}{l}\text { Mini- } \\
\text { Cost }\end{array}$ & $\begin{array}{l}\text { NCT- } \\
\text { Circuits }\end{array}$ & $\begin{array}{l}\text { NCT- } \\
\text { Circuits[14] }\end{array}$ & $\begin{array}{l}\text { NCT- } \\
\text { Circuits[13] }\end{array}$ \\
\hline 0 & 8 & 8 & 8 \\
\hline 1 & 48 & 48 & 48 \\
\hline 2 & 192 & 192 & 192 \\
\hline 3 & 408 & 408 & 408 \\
\hline 4 & 528 & 480 & 480 \\
\hline 5 & 541 & 288 & 288 \\
\hline 6 & 1127 & 592 & 592 \\
\hline 7 & 2413 & 1962 & 2016 \\
\hline 8 & 3566 & 3887 & 4128 \\
\hline 9 & 2430 & 2916 & 2496 \\
\hline 10 & 2545 & 1299 & 672 \\
\hline 11 & 5257 & 3683 & 2880 \\
\hline 12 & 6260 & 7221 & 7488 \\
\hline 13 & 3983 & 6059 & 7488 \\
\hline 14 & 1672 & 1465 & 384 \\
\hline 15 & 3389 & 3562 & 1600 \\
\hline 16 & 4102 & 4201 & 5568 \\
\hline 17 & 1851 & 2049 & 3584 \\
\hline Total & 40320 & 40320 & 40320 \\
\hline Average & 11.459 & 11.769 & 11.983 \\
\hline
\end{tabular}

Table 3: There are number of circuits with minimum length and cost.

\begin{tabular}{|c|c|c|c|c|c|c|c|c|c|c|c|}
\hline $\begin{array}{l}\text { Mini- } \\
\text { Length }\end{array}$ & Cost & \#circuits & $\begin{array}{l}\text { Mini- } \\
\text { Length }\end{array}$ & Cost & \#circuits & $\begin{array}{l}\text { Mini- } \\
\text { Length }\end{array}$ & Cost & \#circuits & $\begin{array}{l}\text { Mini- } \\
\text { Length }\end{array}$ & Cost & \#circuits \\
\hline 1 & 0 & 3 & 4 & 4 & 78 & 5 & 16 & 285 & 7 & 11 & 789 \\
\hline 1 & 1 & 6 & 4 & 5 & 101 & 5 & 17 & 29 & 7 & 12 & 1257 \\
\hline 1 & 5 & 3 & 4 & 6 & 274 & 5 & 18 & 14 & 7 & 13 & 1644 \\
\hline 2 & 0 & 3 & 4 & 7 & 668 & 5 & 19 & 36 & 7 & 14 & 904 \\
\hline 2 & 1 & 24 & 4 & 8 & 280 & 5 & 20 & 12 & 7 & 15 & 1104 \\
\hline 2 & 2 & 24 & 4 & 9 & 96 & 6 & 4 & 45 & 7 & 16 & 1663 \\
\hline 2 & 4 & 3 & 4 & 10 & 310 & 6 & 5 & 168 & 7 & 17 & 1254 \\
\hline 2 & 5 & 18 & 4 & 11 & 384 & 6 & 6 & 129 & 7 & 18 & 380 \\
\hline 2 & 6 & 24 & 4 & 12 & 134 & 6 & 7 & 492 & 7 & 19 & 158 \\
\hline 2 & 9 & 3 & 4 & 13 & 10 & 6 & 8 & 1347 & 7 & 20 & 66 \\
\hline 2 & 10 & 3 & 4 & 14 & 56 & 6 & 9 & 1671 & 7 & 21 & 27 \\
\hline 3 & 0 & 1 & 4 & 15 & 48 & 6 & 10 & 845 & 7 & 22 & 2 \\
\hline 3 & 1 & 18 & 4 & 16 & 6 & 6 & 11 & 2032 & 7 & 23 & 1 \\
\hline 3 & 2 & 117 & 4 & 18 & 1 & 6 & 12 & 2986 & 7 & 24 & 4 \\
\hline 3 & 3 & 51 & 4 & 19 & 1 & 6 & 13 & 2457 & 7 & 25 & 1 \\
\hline 3 & 4 & 12 & 5 & 3 & 75 & 6 & 14 & 839 & 8 & 9 & 3 \\
\hline 3 & 5 & 52 & 5 & 4 & 375 & 6 & 15 & 1376 & 8 & 10 & 2 \\
\hline 3 & 6 & 155 & 5 & 5 & 101 & 6 & 16 & 1588 & 8 & 11 & 17 \\
\hline 3 & 7 & 105 & 5 & 6 & 326 & 6 & 17 & 752 & 8 & 12 & 25 \\
\hline 3 & 8 & 1 & 5 & 7 & 835 & 6 & 18 & 88 & 8 & 13 & 19 \\
\hline 3 & 9 & 25 & 5 & 8 & 1639 & 6 & 19 & 143 & 8 & 14 & 46 \\
\hline 3 & 10 & 55 & 5 & 9 & 523 & 6 & 20 & 75 & 8 & 15 & 89 \\
\hline 3 & 11 & 27 & 5 & 10 & 714 & 6 & 21 & 16 & 8 & 16 & 171 \\
\hline 3 & 13 & 1 & 5 & 11 & 1390 & 7 & 6 & 14 & 8 & 17 & 136 \\
\hline 3 & 14 & 4 & 5 & 12 & 1413 & 7 & 7 & 78 & 8 & 18 & 46 \\
\hline 3 & 15 & 1 & 5 & 13 & 383 & 7 & 8 & 147 & 8 & 19 & 20 \\
\hline 4 & 2 & 51 & 5 & 14 & 273 & 7 & 9 & 465 & 8 & 20 & 2 \\
\hline 4 & 3 & 282 & 5 & 15 & 496 & 7 & 10 & 295 & 8 & 21 & 1 \\
\hline
\end{tabular}

Table 5: There are number of circuits with minimum cost and length.

\begin{tabular}{|c|c|c|c|c|c|c|c|c|c|c|c|}
\hline $\begin{array}{l}\text { Mini- } \\
\text { Cost }\end{array}$ & Length & \#circuits & $\begin{array}{l}\text { Mini- } \\
\text { Cost }\end{array}$ & Length & \#circuits & $\begin{array}{l}\text { Mini- } \\
\text { Cost }\end{array}$ & Length & \#circuits & $\begin{array}{l}\text { Mini- } \\
\text { Cost }\end{array}$ & Length & \#circuits \\
\hline 0 & 1 & 3 & 6 & 7 & 42 & 11 & 4 & 228 & 14 & 9 & 127 \\
\hline 0 & 2 & 3 & 6 & 8 & 2 & 11 & 5 & 1020 & 14 & 10 & 60 \\
\hline 1 & 1 & 6 & 7 & 4 & 511 & 11 & 7 & 1443 & 14 & 12 & 2 \\
\hline 1 & 2 & 24 & 7 & 5 & 916 & 11 & 8 & 441 & 15 & 3 & 1 \\
\hline 1 & 3 & 18 & 7 & 6 & 755 & 11 & 9 & 77 & 15 & 4 & 31 \\
\hline 2 & 3 & 117 & 7 & 8 & 7 & 12 & 4 & 74 & 15 & 6 & 891 \\
\hline 2 & 4 & 51 & 8 & 3 & 1 & 12 & 5 & 812 & 15 & 7 & 1184 \\
\hline
\end{tabular}




\begin{tabular}{|l|l|l||c|c|c||c|c|c||c|c|c|}
\hline 3 & 3 & 51 & 8 & 4 & 174 & 12 & 6 & 2366 & 15 & 8 & 700 \\
\hline 3 & 4 & 282 & 8 & 5 & 1283 & 12 & 7 & 2018 & 15 & 9 & 273 \\
\hline 3 & 5 & 75 & 8 & 6 & 1531 & 12 & 8 & 799 & 15 & 10 & 33 \\
\hline 4 & 2 & 3 & 8 & 7 & 513 & 12 & 9 & 186 & 16 & 4 & 3 \\
\hline 4 & 3 & 12 & 8 & 8 & 56 & 12 & 10 & 5 & 16 & 5 & 122 \\
\hline 4 & 4 & 78 & 8 & 9 & 8 & 13 & 3 & 1 & 16 & 6 & 756 \\
\hline 4 & 5 & 387 & 9 & 2 & 3 & 13 & 4 & 8 & 16 & 7 & 1321 \\
\hline 4 & 6 & 48 & 9 & 3 & 22 & 13 & 5 & 170 & 16 & 8 & 1110 \\
\hline 5 & 1 & 3 & 9 & 4 & 74 & 13 & 6 & 1378 & 16 & 9 & 670 \\
\hline 5 & 2 & 18 & 9 & 5 & 332 & 13 & 7 & 1395 & 16 & 10 & 120 \\
\hline 5 & 3 & 57 & 9 & 6 & 1318 & 13 & 8 & 618 & 17 & 5 & 11 \\
\hline 5 & 4 & 120 & 10 & 2 & 3 & 13 & 9 & 292 & 17 & 6 & 203 \\
\hline 5 & 5 & 140 & 10 & 3 & 43 & 13 & 10 & 109 & 17 & 7 & 424 \\
\hline 5 & 6 & 202 & 10 & 4 & 228 & 13 & 11 & 12 & 17 & 8 & 323 \\
\hline 5 & 7 & 1 & 10 & 5 & 618 & 14 & 3 & 4 & 17 & 9 & 369 \\
\hline 6 & 2 & 18 & 10 & 6 & 908 & 14 & 4 & 42 & 17 & 10 & 353 \\
\hline 6 & 3 & 123 & 10 & 7 & 579 & 14 & 5 & 180 & 17 & 11 & 15 \\
\hline 6 & 4 & 298 & 10 & 8 & 153 & 14 & 6 & 445 & 17 & 12 & 13 \\
\hline 6 & 5 & 429 & 10 & 9 & 13 & 14 & 7 & 545 & & \\
\hline 6 & 6 & 215 & 11 & 3 & 18 & 14 & 8 & 254 & &
\end{tabular}

\section{INTEGRATION OF IRREVERSIBLE BOOLEAN FUNCTIONS IN A REVERSIBLE CIRCUIT}

This section shows that if a set of irreversible Boolean function can be integrated by an even parity permutation that has a cycle representation, then the set of irreversible Boolean functions can be realized by a reversible circuit using NCT library. This provides a concrete realizations for several families of Boolean functions such as $\operatorname{AND}\left(x_{1} \wedge x_{2}\right), O R\left(x_{1} \vee x_{2}\right), \operatorname{NOT}\left(\neg x_{1}\right), X O R\left(x_{1} \oplus x_{2}\right), \operatorname{NAND}\left(x_{1} \uparrow x_{2}\right), \operatorname{NOR}\left(x_{1} \downarrow x_{2}\right)$. For the sake of readability, this will be written shortly as $A N D, O R, N O T, X O R, N A N D$, and NOR respectively.

There exist 8 basics combination of three different irreversible Boolean functions which are $\{\{A N D, O R, N O T\}, \quad\{A N D, N O T, X O R\}, \quad\{A N D, N O T, N O R\}, \quad\{O R, N O T, X O R\}, \quad\{O R, N O T, N A N D\}$, $\{N O T, X O R, N A N D\},\{N O T, X O R, N O R\},\{N O T, N A N D, N O R\}\}$. Each basic combination of three different irreversible Boolean functions has 6 possible permutations, and then we have totally 48 possible combinations of three different irreversible Boolean functions. For example, the reversible circuit to implement the set $\{A N D, O R, N O T\}$ has six different forms, i.e. $(A N D, O R, N O T)$ means that the first output line will be $A N D\left(x_{1} \wedge x_{2}\right)$, the second output line will be $O R\left(x_{1} \vee x_{2}\right)$ and the third line will be $\operatorname{NOT}\left(\neg x_{1}\right)$ which is different from $(A N D, N O T, O R)$ which means that the first output line will be $A N D\left(x_{1} \wedge x_{2}\right)$, the second output line be $\operatorname{NOT}\left(\neg x_{1}\right)$ and the third output line will be $O R\left(x_{1} \vee x_{2}\right)$ as shown in Fig. 10.

Setting the input to $\left(x_{1}, x_{2}, 0\right)$, i.e. the third bit is initialized to 0 , all the possible combinations of three different irreversible Boolean functions can be integrated by an even parity permutation that has a cycle representation and can be realized by reversible circuit as will be shown in Table 6. For example, the combination of $(A N D, O R, N O T)$ can be integrated by a cyclic permutation equal $(1,2)(3,4,5)$ and can be realized by reversible circuit $\left[C_{12}^{3}, C_{13}^{3}, T_{321}^{3}, N_{3}^{3}, C_{12}^{3}\right]$ with best minimum cost equal 8 as shown in Fig. 10.

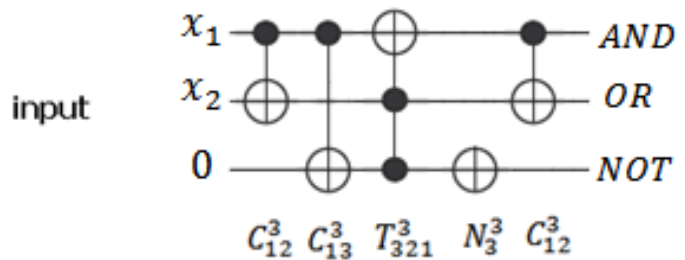

output

Figure 10: The reversible circuit realizes the combination of $(A N D, O R, N O T)$ with the input $\left(x_{1}, x_{2}, 0\right)$.

Setting the input to $\left(x_{1}, x_{2}, 1\right)$, i.e. the third bit is initialized to 1 , all the possible combinations of three different irreversible Boolean functions can be integrated by an even parity permutation that has a cycle representation and can be realized by reversible circuit as will be shown in Table 7. For example, the combination of three different irreversible Boolean functions $(O R, A N D, N O T)$ can be integrated by a cyclic permutation equal $(4,6,5)(7,8)$ and can be realized by reversible circuit $\left[C_{12}^{3}, C_{13}^{3}, T_{321}^{3}, C_{12}^{3}\right]$ with best minimum cost equal 8 as shown in Fig. 11. 


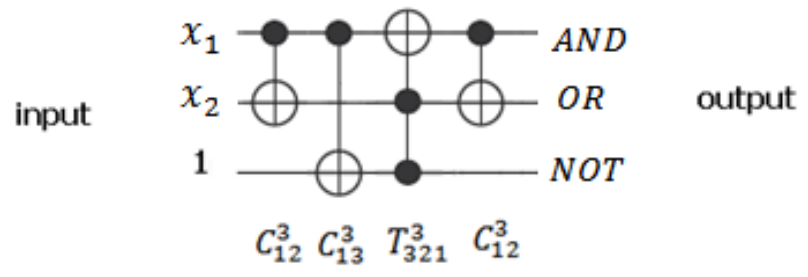

Figure 11: The reversible circuit realizes the combination of $(A N D, O R, N O T)$ with the input $\left(x_{1}, x_{2}, 1\right)$.

All the possible combinations of three different irreversible Boolean functions with the input $\left(x_{1}, x_{2}, 1\right)$ realize the reversible circuits with best minimum cost and length, while all the possible combinations of three different irreversible Boolean functions with different arrangement with the input $\left(x_{1}, x_{2}, 0\right)$ realize the reversible circuits with worst cost and length as shown in Table 8, i.e. setting the input vector to $\left(x_{1}, x_{2}, 1\right)$ gives better results.

Table 6: All the possible Reversible circuits' realizations of Boolean functions combination with input $\left(x_{1}, x_{2}, 0\right)$.

\begin{tabular}{|c|c|c|c|c|}
\hline $\begin{array}{l}\text { All possible } \\
\text { Combination }\end{array}$ & $\begin{array}{c}\text { Cyclic } \\
\text { permutation }\end{array}$ & Reversible circuit & $\begin{array}{l}\text { Quantum } \\
\text { cost }\end{array}$ & $\begin{array}{l}\text { Circuit } \\
\text { Length }\end{array}$ \\
\hline$(A N D, O R, N O T)$ & $(1,2)(3,4,5)$ & {$\left[C_{12}^{3}, C_{13}^{3}, T_{221}^{3}, N_{3}^{3}, C_{12}^{3}\right]$} & 8 & 5 \\
\hline$(A N D, N O T, O R)$ & $(1,3,4)(2,5)(6,7)$ & {$\left[C_{13}^{3}, C_{23}^{3}, C_{32}^{3}, T_{321}^{3}, T_{123}^{3}, N_{2}^{3}\right]$} & 13 & 6 \\
\hline$(O R, A N D, N O T)$ & $(1,2)(3,6)$ & {$\left[N_{3}^{3}, C_{12}^{3}, T_{123}^{3}, T_{321}^{3}, C_{12}^{3}, C_{23}^{3}\right]$} & 13 & 6 \\
\hline$(O R, N O T, A N D)$ & $(1,3,7,6)$ & {$\left[C_{32}^{3}, N_{3}^{3}, T_{123}^{3}, T_{321}^{3}, N_{2}^{3}, T_{132}^{3}, N_{3}^{3}\right]$} & 16 & 7 \\
\hline$(N O T, A N D, O R)$ & $(1,5,2)(3,6)(4,7)$ & {$\left[N_{1}^{3}, C_{12}^{3}, T_{321}^{3}, T_{123}^{3}, C_{32}^{3}, N_{3}^{3}\right]$} & 12 & 6 \\
\hline$(N O T, O R, A N D)$ & $(1,5,3,7,4)$ & {$\left[C_{31}^{3}, N_{3}^{3}, T_{123}^{3}, T_{132}^{3}, N_{1}^{3}, N_{3}^{3}\right]$} & 10 & 6 \\
\hline$(A N D, N O T, X O R)$ & $(1,3,4)(2,7,5)$ & {$\left[N_{2}^{3}, N_{3}^{3}, T_{321}^{3}, N_{3}^{3}, C_{13}^{3}, T_{321}^{3}, N_{3}^{3}, C_{32}^{3}\right]$} & 12 & 8 \\
\hline$(A N D, X O R, N O T)$ & $(1,2)(3,4,7,5)$ & {$\left[C_{13}^{3}, T_{132}^{3}, T_{321}^{3}, N_{3}^{3}\right]$} & 11 & 4 \\
\hline$(N O T, A N D, X O R)$ & $(1,5,2)(3,6,7)$ & {$\left[N_{3}^{3}, C_{31}^{3}, T_{132}^{3}, N_{3}^{3}, C_{23}^{3}, T_{132}^{3}, N_{3}^{3}\right]$} & 12 & 7 \\
\hline$(N O T, X O R, A N D)$ & $(1,5,3,7,2)$ & {$\left[N_{2}^{3}, N_{3}^{3}, T_{132}^{3}, N_{3}^{3}, C_{31}^{3}, T_{123}^{3}, N_{1}^{3}, N_{2}^{3}\right]$} & 11 & 8 \\
\hline$(X O R, A N D, N O T)$ & $(1,2)(3,6,7)$ & {$\left[N_{3}^{3}, T_{321}^{3}, T_{132}^{3}, N_{3}^{3}, C_{23}^{3}, T_{132}^{3}, N_{3}^{3}\right]$} & 15 & 7 \\
\hline$(X O R, N O T, A N D)$ & $(1,3,7,2)$ & {$\left[N_{1}^{3}, N_{3}^{3}, T_{321}^{3}, N_{2}^{3}, T_{132}^{3}, N_{3}^{3}, T_{123}^{3}, N_{1}^{3}, N_{2}^{3}\right]$} & 15 & 9 \\
\hline$(A N D, N O T, N O R)$ & $(1,4,7,5)$ & {$\left[N_{1}^{3}, N_{3}^{3}, T_{321}^{3}, T_{132}^{3}, N_{3}^{3}, T_{123}^{3}, C_{31}^{3}\right]$} & 15 & 7 \\
\hline$(A N D, N O R, N O T)$ & $(1,4,7,5)(2,3)$ & {$\left[C_{12}^{3}, C_{13}^{3}, T_{321}^{3}, N_{2}^{3}, T_{12}^{3}, N_{3}^{3}\right]$} & 12 & 6 \\
\hline (NOT, AND, NOR) & $(1,6,7,3,5)$ & {$\left[N_{2}^{3}, N_{3}^{3}, C_{31}^{3}, T_{123}^{3}, N_{2}^{3}, T_{132}^{3}, N_{3}^{3}\right]$} & 11 & 7 \\
\hline (NOT, NOR, AND) & $(1,7,2,3,5)$ & {$\left[N_{2}^{3}, C_{12}^{3}, C_{31}^{3}, T_{123}^{3}, T_{132}^{3}, N_{1}^{3}\right]$} & 11 & 6 \\
\hline$(N O R, A N D, N O T)$ & $(1,6,5)(2,7,3$ & {$\left[N_{1}^{3}, C_{32}^{3}, T_{123}^{3}, N_{2}^{3}, C_{32}^{3}, C_{31}^{3}, T_{123}^{3}, N_{2}^{3}\right]$} & 13 & 8 \\
\hline$(N O R, N O T, A N D)$ & $(1,7,2,5)$ & {$\left[N_{2}^{3}, N_{3}^{3}, C_{21}^{3}, T_{132}^{3}, N_{3}^{3}, T_{123}^{3}, T_{321}^{3}, N_{2}^{3}\right]$} & 16 & 8 \\
\hline$(O R, N O T, X O R)$ & $(1,3,8)(5,6,7)$ & {$\left[N_{3}^{3}, C_{13}^{3}, T_{321}^{3}, T_{123}^{3}, C_{32}^{3}, N_{3}^{3}\right]$} & 12 & 6 \\
\hline$(O R, X O R, N O T)$ & $(1,2)(3,8)(5,7)$ & {$\left[T_{321}^{3}, N_{3}^{3}, T_{132}^{3}, C_{13}^{3}, T_{321}^{3}\right]$} & 14 & 5 \\
\hline$(N O T, O R, X O R)$ & $(1,5,4)(3,8,7)$ & {$\left[C_{31}^{3}, N_{3}^{3}, T_{132}^{3}, C_{23}^{3}, T_{132}^{3}, N_{1}^{3}, N_{3}^{3}\right]$} & 12 & 7 \\
\hline$(N O T, X O R, O R)$ & $(1,5,4)(2,7)(3,8)$ & {$\left[N_{1}^{3}, C_{12}^{3}, C_{32}^{3}, T_{321}^{3}, N_{3}^{3}, T_{123}^{3}, N_{2}^{3}\right]$} & 12 & 7 \\
\hline$(X O R, O R, N O T)$ & $(1,2)(3,8,5,7)$ & {$\left[N_{3}^{3}, T_{321}^{3}, C_{12}^{3}, C_{23}^{3}, T_{132}^{3}\right]$} & 12 & 5 \\
\hline$(X O R, N O T, O R)$ & $(1,3,8)(2,7)(5,6)$ & {$\left[N_{1}^{3}, T_{132}^{3}, N_{3}^{3}, C_{21}^{3}, C_{12}^{3}, T_{123}^{3}, N_{1}^{3}\right]$} & 12 & 7 \\
\hline$(O R, N O T, N A N D)$ & $(1,4)(3,8)(5,6,7)$ & {$\left[N_{1}^{3}, C_{21}^{3}, C_{13}^{3}, T_{321}^{3}, C_{32}^{3}, N_{3}^{3}, T_{123}^{3}, N_{1}^{3}\right]$} & 13 & 8 \\
\hline$(O R, N A N D, N O T)$ & $(1,4)(3,8)(5,7)$ & {$\left[N_{1}^{3}, T_{123}^{3}, N_{2}^{3}, C_{32}^{3}, T_{321}^{3}, T_{321}^{3}, N_{1}^{3}\right]$} & 16 & 7 \\
\hline (NOT, OR, NAND) & $(1,6)(3,8,7)(4,5)$ & {$\left[N_{1}^{3}, C_{12}^{3}, N_{1}^{3}, T_{321}^{3}, N_{3}^{3}, T_{13}^{3}, N_{1}^{3}, C_{32}^{3}\right]$} & 12 & 8 \\
\hline (NOT, NAND, OR) & $(1,7,2)(3,8)(4,5)$ & {$\left[N_{1}^{3}, N_{2}^{3}, T_{321}^{3}, T_{123}^{3}, C_{12}^{3}, C_{32}^{3}, N_{3}^{3}\right]$} & 10 & 7 \\
\hline$(N A N D, O R, N O T)$ & $(1,6)(3,8,5,5)$ & {$\left[C_{13}^{3}, T_{123}^{3}, N_{1}^{3}, C_{31}^{3}, T_{132}^{3}, C_{13}^{3}\right]$} & 11 & 6 \\
\hline (NAND, NOT, OR) & $(1,7,2)(3,8)(5,6)$ & {$\left[N_{3}^{3}, C_{21}^{3}, C_{31}^{3}, T_{132}^{3}, T_{123}^{3}, C_{31}^{3}\right]$} & 12 & 6 \\
\hline (NOT,XOR, NAND) & $\begin{array}{l}(1,6,7)(3,8)(4,5) \\
\end{array}$ & {$\left[C_{32}^{3}, T_{321}^{3}, N_{3}^{3}, T_{123}^{3}, C_{12}^{3}, N_{1}^{3}\right]$} & $\overline{10}$ & 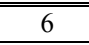 \\
\hline$(N O T, N A N D, X O R)$ & $(1,7)(3,8)(4,5)$ & {$\left[N_{1}^{3}, T_{123}^{3}, N_{1}^{3}, N_{2}^{3}, T_{321}^{3}, C_{21}^{3}, T_{123}^{3}, N_{1}^{3}\right]$} & 14 & 8 \\
\hline$(X O R, N O T, N A N D)$ & $(1,4,7)(3,8)(5,6)$ & {$\left[N_{2}^{3}, N_{3}^{3}, C_{21}^{3}, T_{132}^{3}, T_{123}^{3}, N_{1}^{3}, N_{2}^{3}\right]$} & 10 & 7 \\
\hline$(X O R, N A N D, N O T)$ & $(1,4,5,7)(3,8)$ & {$\left[N_{1}^{3}, T_{123}^{3}, C_{21}^{3}, N_{2}^{3}, C_{22}^{3}, T_{123}^{3}, N_{1}^{3}\right]$} & 11 & 7 \\
\hline$(N A N D, N O T, X O R)$ & $(1,7)(3,8)(5,6)$ & {$\left[N_{1}^{3}, N_{3}^{3}, T_{321}^{3}, T_{132}^{3}, N_{1}^{3}, C_{13}^{3}, T_{321}^{3}, N_{3}^{3}\right]$} & 15 & 8 \\
\hline$(N A N D, X O R, N O T)$ & $(1,6,5,7)(3,8)$ & {$\left[N_{3}^{3}, T_{132}^{3}, C_{31}^{3}, C_{13}^{3}, T_{321}^{3}, N_{3}^{3}\right]$} & 11 & 6 \\
\hline$(N O T, X O R, N O R)$ & $(1,6,5,3,7)$ & {$\left[N_{2}^{3}, N_{3}^{3}, T_{132}^{3}, C_{31}^{3}, N_{3}^{3}, T_{123}^{3}, N_{2}^{3}\right]$} & 10 & 7 \\
\hline (NOT, NOR,XOR) & $(1,7)(2,5)(3,6)$ & {$\left[N_{2}^{3}, T_{123}^{3}, N_{1}^{3}, N_{2}^{3}, C_{32}^{3}, T_{123}^{3}, N_{2}^{3}, T_{321}^{3}\right]$} & 16 & 8 \\
\hline$(X O R, N O T, N O R)$ & $(1,4,3,7)$ & {$\left[N_{1}^{3}, N_{3}^{3}, T_{321}^{3}, T_{132}^{3}, N_{3}^{3}, T_{123}^{3}, N_{1}^{3}\right]$} & 14 & 7 \\
\hline$(X O R, N O R, N O T)$ & $(1,4,7)(3,6)$ & {$\left[N_{2}^{3}, T_{123}^{3}, N_{1}^{3}, T_{321}^{3}, C_{32}^{3}, C_{13}^{3}, T_{321}^{3}\right]$} & 15 & 7 \\
\hline$(N O R, N O T, X O R)$ & $(1,7)(2,5)(3,4)$ & {$\left[N_{2}^{3}, N_{3}^{3}, T_{321}^{3}, N_{3}^{3}, C_{13}^{3}, T_{132}^{3}, T_{321}^{3}, N_{2}^{3}, N_{3}^{3}\right]$} & 16 & 9 \\
\hline$(N O R, X O R, N O T)$ & $(1,6,7)(3,4,5)$ & {$\left[N_{1}^{3}, N_{2}^{3}, T_{321}^{3}, C_{13}^{3}, C_{32}^{3}, T_{321}^{3}\right]$} & 12 & 6 \\
\hline$(N O T, N A N D, N O R)$ & $(1,8,5,3,7)$ & {$\left[N_{1}^{3}, C_{12}^{3}, C_{31}^{3}, T_{123}^{3}, N_{2}^{3}, T_{13}^{3}\right]$} & 12 & 6 \\
\hline
\end{tabular}


Integration of Irreversible Gates in Reversible Circuits Using NCT Library

\begin{tabular}{|l|l|l|c|c|}
\hline$(N O T, N O R, N A N D)$ & $(1,8,7)(2,5)(3,6)$ & {$\left[N_{2}^{3}, T_{123}^{3}, N_{1}^{3}, C_{32}^{3}, T_{32}^{3}, C_{13}^{3}\right]$} & 12 & 6 \\
\hline$(N A N D, N O T, N O R)$ & $(1,8,3,7)$ & {$\left[N_{1}^{3}, N_{3}^{3}, C_{21}^{3}, T_{132}^{3}, N_{3}^{3}, T_{123}^{3}, N_{1}^{3}, T_{321}^{3}\right]$} & 16 & 8 \\
\hline$(N A N D, N O R, N O T)$ & $(1,8,7)(3,6)$ & {$\left[N_{2}^{3}, T_{123}^{3}, N_{1}^{3}, C_{21}^{3}, C_{31}^{3}, C_{13}^{3}\right]$} & 8 & 6 \\
\hline$(N O R, N O T, N A N D)$ & $(1,8,7)(2,5)(3,4)$ & {$\left[C_{23}^{3}, N_{2}^{3}, T_{132}^{3}, C_{23}^{3}, C_{21}^{3}, N_{2}^{3}, T_{132}^{3}\right]$} & 13 & 7 \\
\hline$(N O R, N A N D, N O T)$ & $(1,8,7)(3,4,5)$ & {$\left[N_{1}^{3}, T_{123}^{3}, N_{2}^{3}, C_{32}^{3}, C_{31}^{3}, T_{123}^{3}\right]$} & 12 & 6 \\
\hline
\end{tabular}

Table 7: All the possible Reversible circuits' realizations of Boolean functions combination with $\operatorname{input}\left(x_{1}, x_{2}, 1\right)$.

\begin{tabular}{|c|c|c|c|c|}
\hline $\begin{array}{l}\text { All possible } \\
\text { Combination }\end{array}$ & Cyclic permutation & Reversible circuit & $\begin{array}{l}\text { Quantum } \\
\text { cost }\end{array}$ & $\begin{array}{l}\text { Circuit } \\
\text { Length }\end{array}$ \\
\hline$(A N D, O R, N O T)$ & $(3,6)(7,8)$ & {$\left[C_{23}^{3}, C_{21}^{3}, T_{132}^{3}, T_{123}^{3}, C_{21}^{3}\right]$} & 11 & $\overline{5}$ \\
\hline$(A N D, N O T, O R)$ & $(2,3,8,6)$ & {$\left[N_{1}^{3}, C_{32}^{3}, T_{123}^{3}, T_{321}^{3}, T_{132}^{3}, N_{1}^{3}\right]$} & 15 & 6 \\
\hline$(O R, A N D, N O T)$ & $(4,6,5)(7,8)$ & {$\left[C_{12}^{3}, C_{13}^{3}, T_{321}^{3}, C_{12}^{3}\right]$} & 8 & 4 \\
\hline$(O R, N O T, A N D)$ & $(2,3)(4,7)(5,8,6)$ & {$\left[N_{1}^{3}, N_{3}^{3}, C_{21}^{3}, C_{32}^{3}, T_{123}^{3}, N_{1}^{3}, C_{12}^{3}, T_{321}^{3}, N_{2}^{3}\right]$} & 13 & 7 \\
\hline$(N O T, A N D, O R)$ & $(2,5,8,4,6)$ & {$\left[N_{2}^{3}, C_{31}^{3}, T_{123}^{3}, N_{2}^{3}, T_{132}^{3}\right]$} & 11 & 5 \\
\hline$(N O T, O R, A N D)$ & $(2,5)(3,6)(4,7,8)$ & {$\left[N_{1}^{3}, C_{12}^{3}, C_{23}^{3}, T_{321}^{3}, T_{123}^{3}, C_{32}^{3}, N_{3}^{3}\right]$} & 13 & 7 \\
\hline$\overline{(A N D, N O T, X O R)}$ & $(2,3,6)(5,8)$ & {$\left[C_{23}^{3}, C_{12}^{3}, T_{321}^{3}, C_{32}^{3}, C_{23}^{3}\right]$} & 7 & 5 \\
\hline$(A N D, X O R, N O T)$ & $(3,6)(5,8)$ & {$\left[N_{3}^{3}, T_{321}^{3}, C_{12}^{3}, C_{13}^{3}, T_{321}^{3}, N_{3}^{3}\right]$} & 12 & 6 \\
\hline$(N O T, A N D, X O R)$ & $(2,5,4,6)(3,8)$ & {$\left[C_{31}^{3}, C_{13}^{3}, C_{23}^{3}, T_{132}^{3}, T_{321}^{3}\right]$} & 13 & 5 \\
\hline$(N O T, X O R, A N D)$ & $(2,5,4,7,8)(3,6)$ & {$\left[N_{3}^{3}, T_{321}^{3}, N_{2}^{3}, C_{12}^{3}, T_{321}^{3}, T_{123}^{3}, N_{1}^{3}, N_{2}^{3}\right]$} & 16 & 8 \\
\hline$(X O R, A N D, N O T)$ & $(3,8)(4,6,5)$ & {$\left[C_{13}^{3}, C_{21}^{3}, T_{132}^{3}, T_{321}^{3}, T_{123}^{3}\right]$} & 17 & 5 \\
\hline$(X O R, N O T, A N D)$ & $(2,3,4,7,8)(5,6)$ & {$\left[N_{1}^{3}, T_{321}^{3}, N_{2}^{3}, T_{132}^{3}, N_{3}^{3}, T_{123}^{3}, N_{1}^{3}, N_{2}^{3}\right]$} & 15 & 8 \\
\hline$(A N D, N O T, N O R)$ & $(1,6)(2,4,3)(5,8)$ & {$\left[N_{1}^{3}, N_{3}^{3}, C_{21}^{3}, C_{12}^{3}, C_{32}^{3}, T_{123}^{3}, T_{321}^{3}\right]$} & 13 & 7 \\
\hline$(A N D, N O R, N O T)$ & $(1,6)(2,4)(5,8)$ & {$\left[N_{2}^{3}, N_{3}^{3}, T_{123}^{3}, T_{321}^{3}, N_{3}^{3}, C_{32}^{3}, T_{123}^{3}, N_{2}^{3}\right]$} & 16 & 8 \\
\hline$(N O T, A N D, N O R)$ & $(1,2,6)(3,8)(4,5)$ & {$\left[N_{1}^{3}, N_{3}^{3}, C_{12}^{3}, T_{321}^{3}, T_{123}^{3}, C_{32}^{3}\right]$} & 12 & 6 \\
\hline$(N O T, N O R, A N D)$ & $(1,4,2,6)(3,8)$ & {$\left[N_{1}^{3}, N_{3}^{3}, T_{123}^{3}, T_{132}^{3}, C_{31}^{3}, C_{13}^{3}\right]$} & 10 & 6 \\
\hline$(N O R, A N D, N O T)$ & $(1,6)(2,7,8)(4,5)$ & {$\left[N_{3}^{3}, T_{321}^{3}, T_{123}^{3}, N_{1}^{3}, C_{12}^{3}, C_{32}^{3}\right]$} & 12 & 6 \\
\hline$(N O R, N O T, A N D)$ & $(1,6)(2,7,8)(3,4)$ & {$\left[N_{3}^{3}, C_{23}^{3}, T_{132}^{3}, N_{1}^{3}, N_{3}^{3}, C_{23}^{3}, C_{21}^{3}, T_{132}^{3}, N_{3}^{3}\right]$} & 13 & 9 \\
\hline$(O R, N O T, X O R)$ & $(2,3)(4,8,5)$ & {$\left[C_{23}^{3}, C_{12}^{3}, C_{32}^{3}, C_{23}^{3}, T_{321}^{3}\right]$} & 9 & 5 \\
\hline$(O R, X O R, N O T)$ & $(4,8,5)(6,7)$ & {$\left[C_{12}^{3}, C_{13}^{3}, T_{321}^{3}\right]$} & 7 & 3 \\
\hline$(N O T, O R, X O R)$ & $(2,5)(3,6,4,8)$ & {$\left[C_{13}^{3}, C_{21}^{3}, C_{31}^{3}, C_{13}^{3}, T_{132}^{3}, T_{321}^{3}\right]$} & 14 & 6 \\
\hline$(N O T, X O R, O R)$ & $(2,5,6,4,8)$ & {$\left[N_{2}^{3}, T_{132}^{3}, C_{31}^{3}, T_{123}^{3}, N_{2}^{3}\right]$} & 10 & 5 \\
\hline$(X O R, O R, N O T)$ & $(3,4,8)(6,7)$ & {$\left[T_{321}^{3}, C_{23}^{3}, T_{132}^{3}, T_{123}^{3}\right]$} & 14 & 4 \\
\hline$(X O R, N O T, O R)$ & $(2,3,4,8)$ & {$\left[N_{1}^{3}, T_{321}^{3}, T_{132}^{3}, T_{123}^{3}, N_{1}^{3}\right]$} & 13 & 5 \\
\hline$(O R, N O T, N A N D)$ & $(2,4,8,5)$ & {$\left[N_{2}^{3}, C_{32}^{3}, T_{123}^{3}, T_{321}^{3}, N_{2}^{3}, T_{132}^{3}\right]$} & 16 & 6 \\
\hline$(O R, N A N D, N O T)$ & $(2,4,8,5)(6,7)$ & {$\left[N_{2}^{3}, C_{13}^{3}, T_{321}^{3}, N_{2}^{3}, C_{12}^{3}, T_{321}^{3}\right]$} & 12 & 6 \\
\hline$(N O T, O R, N A N D)$ & $(2,6,4,8,3)$ & {$\left[N_{1}^{3}, C_{31}^{3}, T_{123}^{3}, T_{132}^{3}, N_{1}^{3}\right]$} & 10 & 5 \\
\hline$(N O T, N A N D, O R)$ & $(2,7,6,4,8)$ & {$\left[C_{32}^{3}, C_{31}^{3}, T_{123}^{3}, T_{132}^{3}\right]$} & 11 & 4 \\
\hline$(N A N D, O R, N O T)$ & $(2,6,7)(3,4,8)$ & {$\left[C_{23}^{3}, T_{132}^{3}, C_{21}^{3}, C_{31}^{3}, T_{123}^{3}\right]$} & 13 & 5 \\
\hline$(N A N D, N O T, O R)$ & $(2,7,4,8)$ & {$\left[C_{32}^{3}, C_{31}^{3}, T_{123}^{3}, T_{321}^{3}, T_{132}^{3}\right]$} & 16 & 5 \\
\hline$(N O T, X O R, N A N D)$ & $(1,2,6,4,8)$ & {$\left[N_{1}^{3}, N_{2}^{3}, C_{31}^{3}, T_{132}^{3}, T_{123}^{3}, N_{1}^{3}, N_{2}^{3}\right]$} & 10 & 7 \\
\hline$(N O T, N A N D, X O R)$ & $(1,6,4,8)(2,7)$ & {$\left[C_{12}^{3}, N_{1}^{3}, C_{23}^{3}, T_{321}^{3}, T_{132}^{3}, N_{3}^{3}\right]$} & 11 & 6 \\
\hline$(X O R, N O T, N A N D)$ & $(1,2,4,8)$ & {$\left[N_{1}^{3}, T_{321}^{3}, N_{1}^{3}, T_{132}^{3}, T_{123}^{3}, N_{1}^{3}, N_{2}^{3}\right]$} & 14 & 7 \\
\hline$(X O R, N A N D, N O T)$ & $(1,2,4,8)(6,7)$ & {$\left[T_{321}^{3}, C_{12}^{3}, C_{32}^{3}, C_{23}^{3}, T_{132}^{3}, N_{3}^{3}\right]$} & 13 & 6 \\
\hline$(N A N D, N O T, X O R)$ & $(1,4,8)(2,7)$ & {$\left[N_{1}^{3}, C_{21}^{3}, C_{12}^{3}, N_{1}^{3}, C_{13}^{3}, T_{321}^{3}, N_{3}^{3}\right]$} & 8 & 7 \\
\hline$(N A N D, X O R, N O T)$ & $(1,4,8)(2,6,7)$ & {$\left[N_{2}^{3}, N_{3}^{3}, T_{321}^{3}, C_{13}^{3}, N_{1}^{3}, C_{12}^{3}, T_{321}^{3}, N_{3}^{3}\right]$} & 12 & 8 \\
\hline$(N O T, X O R, N O R)$ & $(1,8)(2,6,3)(4,7)$ & {$\left[N_{2}^{3}, C_{31}^{3}, N_{3}^{3}, T_{123}^{3}, C_{12}^{3}, T_{321}^{3}\right]$} & 10 & 6 \\
\hline$(N O T, N O R, X O R)$ & $(1,8)(2,7,4,6)$ & {$\left[N_{3}^{3}, C_{12}^{3}, T_{321}^{3}, C_{23}^{3}, T_{132}^{3}, N_{1}^{3}, C_{12}^{3}\right]$} & 12 & 7 \\
\hline$(X O R, N O T, N O R)$ & $(1,8)(2,4,7)(5,6)$ & {$\left[N_{1}^{3}, N_{2}^{3}, N_{3}^{3}, T_{132}^{3}, T_{123}^{3}, C_{21}^{3}, C_{12}^{3}\right]$} & 10 & 7 \\
\hline$(X O R, N O R, N O T)$ & $(1,8)(2,4,6,5)$ & {$\left[N_{1}^{3}, C_{21}^{3}, T_{132}^{3}, C_{31}^{3}, N_{3}^{3}, C_{23}^{3}, T_{132}^{3}\right]$} & 12 & 7 \\
\hline$(N O R, N O T, X O R)$ & $(1,8)(2,7,6)$ & {$\left[N_{2}^{3}, C_{12}^{3}, C_{23}^{3}, C_{21}^{3}, C_{12}^{3}, T_{321}^{3}, N_{2}^{3}\right]$} & 9 & 7 \\
\hline$(N O R, X O R, N O T)$ & $(1,8)(2,6,3)$ & {$\left[N_{1}^{3}, C_{31}^{3}, C_{12}^{3}, C_{13}^{3}, N_{1}^{3}, T_{321}^{3}\right]$} & 8 & 6 \\
\hline$(N O T, N A N D, N O R)$ & $(1,2,8)(3,6)(4,7)$ & {$\left[N_{1}^{3}, N_{2}^{3}, N_{3}^{3}, T_{321}^{3}, T_{123}^{3}, C_{12}^{3}, C_{32}^{3}\right]$} & 10 & 7 \\
\hline (NOT, NOR, NAND) & $(1,4,6,2,8)$ & {$\left[N_{1}^{3}, N_{2}^{3}, C_{31}^{3}, T_{123}^{3}, C_{32}^{3}, T_{123}^{3}, N_{1}^{3}, N_{2}^{3}\right]$} & 12 & 8 \\
\hline$(N A N D, N O T, N O R)$ & $(1,2,8)(4,7)(5,6)$ & {$\left[C_{21}^{3}, C_{31}^{3}, T_{132}^{3}, T_{123}^{3}, C_{31}^{3}, N_{3}^{3}\right]$} & 12 & 6 \\
\hline$(N A N D, N O R, N O T)$ & $(1,2,8)(4,6,5)$ & {$\left[N_{2}^{3}, T_{123}^{3}, N_{1}^{3}, C_{32}^{3}, C_{31}^{3}, T_{123}^{3}, N_{1}^{3}, N_{2}^{3}\right]$} & 12 & 8 \\
\hline$(N O R, N O T, N A N D)$ & $(1,6,2,8)$ & {$\left[N_{1}^{3}, T_{321}^{3}, N_{2}^{3}, T_{132}^{3}, T_{123}^{3}, N_{1}^{3}, N_{2}^{3}, C_{31}^{3}\right]$} & 15 & 8 \\
\hline$(N O R, N A N D, N O T)$ & $(1,2,8)(3,6)$ & {$\left[N_{1}^{3}, T_{123}^{3}, C_{32}^{3}, N_{3}^{3}, C_{31}^{3}, C_{13}^{3}\right]$} & 8 & 6 \\
\hline
\end{tabular}


Table 8: Comparison between the possible combinations of Boolean functions which realize the reversible circuits with best minimum cost and length while the inputs $\left(x_{1}, x_{2}, 0\right)$ and $\left(x_{1}, x_{2}, 1\right)$.

\begin{tabular}{|c|c|c|c|}
\hline \multicolumn{4}{|c|}{ Input $\left(x_{1}, x_{2}, 0\right)$} \\
\hline $\begin{array}{l}\text { Basic Possible } \\
\text { Combinations }\end{array}$ & $\begin{array}{l}\text { Reversible } \\
\text { Circuits implementation }\end{array}$ & Mini-Cost & Length \\
\hline$(A N D, O R, N O T)$ & {$\left[C_{12}^{3}, C_{13}^{3}, T_{321}^{3}, N_{3}^{3}, C_{12}^{3}\right]$} & 8 & 5 \\
\hline$(A N D, X O R, N O T)$ & {$\left[C_{13}^{3}, T_{132}^{3}, T_{321}^{3}, N_{3}^{3}\right]$} & 11 & 4 \\
\hline$(N O T, N O R, A N D)$ & {$\left[N_{2}^{3}, C_{12}^{3}, C_{31}^{3}, T_{123}^{3}, T_{132}^{3}, N_{1}^{3}\right]$} & 11 & 6 \\
\hline$(X O R, O R, N O T)$ & {$\left[N_{3}^{3}, T_{321}^{3}, C_{12}^{3}, C_{23}^{3}, T_{132}^{3}\right]$} & 12 & 5 \\
\hline$(N O T, N A N D, O R)$ & {$\left[N_{1}^{3}, N_{2}^{3}, T_{321}^{3}, T_{123}^{3}, C_{12}^{3}, C_{32}^{3}, N_{3}^{3}\right]$} & 10 & 7 \\
\hline (XOR, NOT, NAND) & {$\left[N_{2}^{3}, N_{3}^{3}, C_{21}^{3}, T_{132}^{3}, T_{123}^{3}, N_{1}^{3}, N_{2}^{3}\right]$} & 10 & 7 \\
\hline (NOT, XOR, NOR) & {$\left[N_{2}^{3}, N_{3}^{3}, T_{132}^{3}, C_{31}^{3}, N_{3}^{3}, T_{123}^{3}, N_{2}^{3}\right]$} & 10 & 7 \\
\hline$(N A N D, N O R, N O T)$ & {$\left[N_{2}^{3}, T_{123}^{3}, N_{1}^{3}, C_{21}^{3}, C_{31}^{3}, C_{13}^{3}\right]$} & 8 & 6 \\
\hline \multicolumn{4}{|c|}{ Input $\left(x_{1}, x_{2}, 1\right)$} \\
\hline $\begin{array}{l}\text { Basic Possible } \\
\text { Combinations }\end{array}$ & $\begin{array}{l}\text { Reversible } \\
\text { Circuits implementation }\end{array}$ & Mini-Cost & Length \\
\hline$(O R, A N D, N O T)$ & {$\left[C_{12}^{3}, C_{13}^{3}, T_{321}^{3}, C_{12}^{3}\right]$} & 8 & 4 \\
\hline$(A N D, N O T, X O R)$ & {$\left[C_{23}^{3}, C_{12}^{3}, T_{321}^{3}, C_{32}^{3}, C_{23}^{3}\right]$} & 7 & 5 \\
\hline$(N O T, N O R, A N D)$ & {$\left[N_{1}^{3}, N_{3}^{3}, T_{123}^{3}, T_{132}^{3}, C_{31}^{3}, C_{13}^{3}\right]$} & 10 & 6 \\
\hline$(O R, X O R, N O T)$ & {$\left[C_{12}^{3}, C_{13}^{3}, T_{321}^{3}\right]$} & 7 & 3 \\
\hline (NOT, OR, NAND) & {$\left[N_{1}^{3}, C_{31}^{3}, T_{123}^{3}, T_{132}^{3}, N_{1}^{3}\right]$} & 10 & 5 \\
\hline$(N A N D, N O T, X O R)$ & {$\left[N_{1}^{3}, C_{21}^{3}, C_{12}^{3}, N_{1}^{3}, C_{13}^{3}, T_{321}^{3}, N_{3}^{3}\right]$} & 8 & 7 \\
\hline$(N O R, X O R, N O T)$ & {$\left[N_{1}^{3}, C_{31}^{3}, C_{12}^{3}, C_{13}^{3}, N_{1}^{3}, T_{321}^{3}\right]$} & 8 & 6 \\
\hline$(N O R, N A N D, N O T)$ & {$\left[N_{1}^{3}, T_{123}^{3}, C_{32}^{3}, N_{3}^{3}, C_{31}^{3}, C_{13}^{3}\right]$} & 8 & 6 \\
\hline
\end{tabular}

\section{CONCLUSION}

By reducing the representation of the reversible circuit synthesis problem to permutation group, Schreier-Sims Algorithm for the strong generating set-finding problem is used to synthesize reversible circuits with minimal length. Applying the proposed optimization rules on the synthesized circuits gives better quantum cost to be 11.459 better than other results shown in the literature. The minimal length of a reversible circuit is obtained by using the main NCT library which the minimal quantum cost is obtained from using a sub library from the main NCT library.

Digital logic design is a well established area of research where classical Boolean functions in used in the construction process. Classical Boolean functions are mainly irreversible and cannot be used directly in the reversible circuits' synthesis. It was shown how to integrate any three Boolean functions in a single reversible circuit using the NCT library. It was shown that the order of the Boolean function in the output vector affects the efficiency of the circuit. There are two ways to initialize the input vector, $\left(x_{1}, x_{2}, 0\right)$ and $\left(x_{1}, x_{2}, 1\right)$. It was shown that initializing the input vector to $\left(x_{1}, x_{2}, 1\right)$ gives better results with respect to the length and the quantum cost of the circuit.

\section{ACKNOWLEDGEMENTS}

We would like to thank Dr Ragab Omar, Department of Mathematics, Faculty of Science, Damanhour University, Egypt for their help and encouragement.

\section{References}

[1]. C. Bennett, Logical reversibility of computation. IBM Journal of Research and Development, 17(6):525-532 (1973).

[2]. E. Fredkin, and T. Toffoli, Conservative logic, International Journal of Theoretical Physics, 21:219-253 (1982).

[3]. J. Gruska, Quantum computing, McGraw-Hill, London (1999).

[4]. M. Nielsen, and I. Chuang, Quantum computation and quantum information, Cambridge University Press, Cambridge, United Kingdom (2000).

[5]. A. De Vos, B. Desoete, A. Adamski, P. Pietrzak, M. Sibinski, and T. Widerski, Design of reversible logic circuits by means of control gates, Proc. 10th International Workshop on Integrated Circuit Design, Power and Timing Modeling, Optimization and Simulation, pages 255-264 (2000).

[6]. A. De Vos, B. Desoete, F. Janiak, and A. Nogawski, Control gates as building blocks for reversible computers, Proc. 11th International Workshop on Power and Timing Modeling, Optimization and Simulation, pages 9201 --9210 (2001).

[7]. T. Toffoli. Reversible computing. InW. de Bakker, and J. van Leeuwen, editors, Automata, Languages and Programming, page 632. Springer, New York, 1980. Technical Memo MIT/LCS/TM-151, MIT Lab for Computer Science.

[8]. D. Simon, On the power of quantum computation, Proc. 35th Annual Symposium on Foundations of Computer Science, pages 116123 (1994).

[9]. A. De Vos, B. Raa, and L. Storme, Generating the group of reversible logic gates, Journal of Physics A: Mathematical and General, 35(33): 7063-7078 (2002).

[10]. L. Storme, A. De Vos, and G. Jacobs, Group theoretical aspects of reversible logic gates, Journal of Universal Computer Science, 5(5): 307-321 (1999).

[11]. A. De Vos, and Y. V. Rentergem, From group theory to reversible computers. International Journal of Unconventional Computing, 4(1): 79-88 (2008). 
[12]. A. De Vos and S. De Baerdemacker, Symmetry groups for the decomposition of reversible computers, quantum computers, and computers in between. Symmetry, 3(2): 305-324 (2011).

[13]. G. Yang, X. Song, W. N.N. Hung, M. A. Perkowski, and C.-J. Seo, Synthesis of reversible circuits with minimal costs, CALCOLO, 45:193-206, 2008.

[14]. A. Younes, Tight bounds on the synthesis of 3-bit reversible circuits: NFT library, arXiv:1304.5804v2 (2013).

[15]. A. Seress, Permutation group algorithms, Cambridge University Press (2002).

[16]. M.I. Kargapolov, and Ju.I. Merzljakov, Fundamentals of the theory of groups, Berlin: Springer (1979).

[17]. J.D. Dixon, and B. Mortimer, Permutation groups, New York: Springer (1996).

[18]. A. Younes, A single universal n-bit gate for reversible circuit synthesis, arXiv: 1306.1254v1 (2013).

[19]. A. Barenco, C. H. Bennett, R. Cleve, D. P. DiVincenzo, N. Margolus, P. Shor, T. Sleator, J. A. Smolin, and H. Weinfurter, Elementary gates for quantum computation, Physical Review A, 52(5):3457- 3467 (1995).

[20]. J.A. Smolin and D.P. DiVincenzo, Five two-bit quantum gates are sufficient to implement the quantum Fredkin gate, Physical Review A, 53, 2855-2856 (1996).

[21]. D. Maslov, Reversible logic synthesis benchmarks, [Online], Available: http://www.cs.uvic.ca/ dmaslov/.

[22]. The GAP Group. GAP - Groups, algorithms, and programming, Version 4.6.3; 2013. Available: http://www.gap-system.org 